\section{Closed-form solutions of transient electro-osmotic flow driven by AC electric field in a microannulus}

Shaowei Wang ${ }^{1,2^{*}}$ and Moli Zhao ${ }^{1,2}$

\begin{tabular}{l}
\hline${ }^{*}$ Correspondence: \\
shaoweiwang@sdu.edu.cn \\
'Department of Engineering \\
Mechanics, School of Civil \\
Engineering, Shandong University, \\
Jinan, 250061, P.R. China \\
${ }^{2}$ Geotechnical and Structural \\
Engineering Research Center, \\
Shandong University, Jinan, 250061, \\
P.R. China
\end{tabular}

"Correspondence:

shaoweiwang@sdu.edu.cn

Department of Engineering

Engineering, Shandong University, Jinan, 250061, P.R. China

Engineering Research Center, P.R. China

\section{Springer} in any medium, provided the original work is properly credited.

\begin{abstract}
The time-periodic electro-osmotic flow of Newtonian fluids through a microannulus is studied in the Debye-Hückel approximation. Analytical series solutions for velocity and flow rate are presented with the help of an integral transform. The expression for the distribution of the velocity profile consists of a time-dependent oscillating part and a time-dependent generating or transient one, and the normalized velocity function is independent of the Reynolds number, which is very different from previous results. Then the effects of the electrokinetic width $K$, the wall zeta potential ratio $\beta$, and the frequency of applied external electric field $\omega$ on the distribution of the velocity profiles and flow rates are discussed numerically and theoretically. Some new physical and chemical phenomena are found theoretically. We point out that the electro-osmotic flow driven by an alternating electric field is not periodic in time, but quasi-periodic. There is a phase shift between voltage and flow, which is only dependent on the frequency of the external electric field.
\end{abstract}

Keywords: transient flow; electro-osmosis flow; integral transform; velocity distribution

\section{Introduction}

When an electric field is applied to the fluids in a channel, the walls of which are charged, the migration of the ions present in excess in the double layer induces the motion of the bulk solution due to viscous drag. This phenomenon provides an attractive means of manipulating liquids in microdevices, and it has been widely used in different microdevices and for various applications, such as microfractionation [1, 2], electrophoresis [3], and microspray generation systems [4].

Time-periodic electro-osmotic flow is also known as AC electro-osmosis, and it is driven by an alternating electric field. It is very important for biotechnology and separation science. Recently, various studies analyzed the time-periodic electro-osmotic flow theory and modeling in different geometry. Dutta and Beskok [5] were among the early researchers who analytically investigated the time-periodic electro-osmotic flow between two parallel plates, illustrating interesting similarities or dissimilarities with the Stokes second problem. Based on the method proposed by Dutta and Beskok, many researchers studied timeperiodic electro-osmotic flows through microchannels, and some new results are given. General solutions were developed by Xuan and Li [6] for direct current and alternating

(C) 2014 Wang and Zhao; licensee Springer. This is an Open Access article distributed under the terms of the Creative Commons Attribution License (http://creativecommons.org/licenses/by/4.0), which permits unrestricted use, distribution, and reproduction 
current electro-osmotic flows in microfluidic channels with arbitrary cross-sectional geometry and arbitrary distribution of wall charge. Jian et al. and his colleagues investigated the flow behavior of time-periodic electro-osmosis in a cylindrical microannulus $[7,8]$.

Unfortunately, due to the incorrect critical assumption of the form of velocity distribution, the results given in these researches are not correct, and some very important physical phenomena have not been found theoretically. In their researches, these authors believed that the velocity profiles will be oscillatory, and they assumed that these oscillations are instantaneous responses of the externally applied electric fields, i.e., they have the same frequency. True, the electro-osmotic flows should really be generated by the applied time-periodic electric fields, and the flows may be time periodic. But, as we know, there is a phase difference between phase voltage and phase current, and the flow in the microchannel should need some time to start. In other words, there is a phase difference between the applied electric fields and the electro-osmotic flows. On the other hand, on the basis of the aforementioned 'assumption', the obtained analytical solutions of velocities are represented as complex functions, which is unreasonable in physics. So, as a result, the solutions given in these research papers are, generally speaking, incorrect.

In fact, the phase shift between the applied electric field and the flow response has been proved by Nayak [9], as well as some other researchers [10]. The steady/unsteady electroosmotic flow in an infinitely extended cylindrical channel with diameters ranging from 10 to $100 \mathrm{~nm}$ has been investigated by Nayak [9], and the degree of the phase shift between the velocity field and the applied electric field is found numerically. Using the backwards-Euler time stepping numerical method, Luo [10] clarified the relationship between the changes in the axial-flow velocity and the intensity of the applied electric field. Erickson and Li [11] developed the analytical solution for the AC electro-osmotic flow through a rectangular microchannel for the case of a sinusoidal applied electric field. Shilov et al. [12] discussed the mechanisms for different times after the application of the electrical field according to the relationship between the dipole moment and the electrophoretic mobility.

The aim of the present paper is to present the analytical solutions for the time-periodic electro-osmotic flow of Newtonian fluids through a microannulus. Analytical solutions are rare. Not only do they represent electro-osmotic flows through fundamental crosssectional shapes but they also serve as standards for asymptotic and fully numerical methods. Most important of all, some new physical and chemical phenomena can be found from the analytical solutions.

\section{Governing equations}

The motions of an ionized, incompressible Newtonian fluid with electro-osmotic body forces are governed by the following Navier-Stokes equation:

$$
\rho \frac{D \mathbf{V}}{D t}=-\nabla P+\mu \nabla^{2} \mathbf{V}+\rho_{\mathrm{e}} \mathbf{E},
$$

where $P$ is the pressure, $\rho$ is the fluid density, $\mu$ is the dynamic viscosity, and the tensor $\mathbf{V}$ is a divergence-free velocity field, i.e., $\nabla \cdot \mathbf{V}=0$ subject to the non-slip boundary conditions on the walls, $\mathbf{E}=\mathbf{E}_{0} f(t)$ is the externally applied electric field, and $\rho_{\mathrm{e}}$ is the electric charge density, which can be expressed by a potential distribution $\psi$; we have

$$
\nabla^{2} \psi=-\frac{\rho_{\mathrm{e}}}{\varepsilon}
$$


and

$$
\rho_{\mathrm{e}}(r)=-2 n_{0} z_{\mathrm{v}} e \sinh \left[\frac{z_{\mathrm{v}} e \psi(r)}{k_{\mathrm{b}} T}\right]
$$

here $n_{0}$ is the bulk electrolyte concentration of a binary electrolyte dissociating into cations and anions of valence $z_{\mathrm{v}}, e$ is the electron charge, $k_{\mathrm{b}}$ is the Boltzmann constant, and $T$ is the absolute temperature.

In the present study, we assume the surface potential is small enough, then with the help of Debye-Hückel approximation and cylindrical coordinate system $(r, \theta, z)$, Eq. (2) is linearized to

$$
\frac{1}{r} \frac{\partial}{\partial r}\left(r \frac{\partial \psi}{\partial r}\right)=\kappa^{2} \psi
$$

where $\kappa^{2}=2 z_{\mathrm{v}}^{2} e^{2} n_{0} / \varepsilon k_{\mathrm{b}} T$ is the Debye-Hückel parameter and $1 / \kappa$ means the Debye length.

Because of the effect of the electric field, the fluid in the capillary will flow along the axis direction. Neglecting the pressure gradient along the axis, the Cauchy momentum equation in cylindrical coordinate system with $\mathrm{AC}$ electric field can be expressed as

$$
\rho \frac{\partial u}{\partial t}=\mu \frac{1}{r} \frac{\partial}{\partial r}\left(r \frac{\partial u}{\partial r}\right)+\rho_{\mathrm{e}}(r) E_{0} \cos (\omega t)
$$

where $u=u(r, t)$ is the axial velocity, $t$ is time, and $E_{0} \cos (\omega t)$ is AC electric field, $E_{0}$ is the magnitude, and $\omega$ is the frequency of the unsteady external electric field $\mathbf{E}$.

In the present research, the geometric shape of the microchannel is considered, as shown in Figure 1. An electrolyte fluid is flowing unsteadily in the annular region between two uniform coaxial circular cylinders with inner radius $R_{\mathrm{i}}=\alpha R(0<\alpha<1)$ and outer radius $R_{\mathrm{o}}=R$. The chemical interaction of the electrolyte liquid and solid wall generates an electric double layer (EDL), a very thin charged liquid layer at the solid-liquid interface. The outer and inner wall zeta potentials are $\psi_{\mathrm{o}}$ and $\psi_{\mathrm{i}}$, respectively. Here, $\psi_{\mathrm{o}}$ and $\psi_{\mathrm{i}}$ are small enough, so that the Debye-Hückel linearization approximation is available.

Define the following dimensionless variables:

$$
r^{*}=\frac{r}{R}, \quad t^{*}=\frac{\mu t}{\rho R^{2}}, \quad \omega^{*}=\frac{\rho R^{2} \omega}{\mu}, \quad \psi^{*}=\frac{z_{\mathrm{v}} e \psi}{k_{\mathrm{b}} T}, \quad u^{*}=\frac{u}{U_{\mathrm{eo}}},
$$
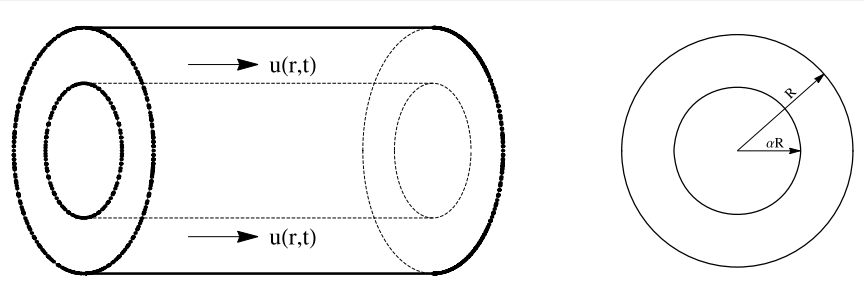

Figure 1 The section of semicircular channel with different constant zeta potentials on the boundaries. 
here $U_{\mathrm{eo}}=-\varepsilon k_{\mathrm{b}} T E_{0} / \mu z_{\mathrm{v}} e$. Substituting the above dimensionless variables into (4) and (5) yields the governing equation for the potential distribution with boundary conditions

$$
\begin{aligned}
& \frac{1}{r} \frac{\partial}{\partial r}\left(r \frac{\partial \psi}{\partial r}\right)=K^{2} \psi, \\
& \psi=\psi_{\mathrm{o}}^{*}, \quad r=1, \\
& \psi=\psi_{\mathrm{i}}^{*}, \quad r=\alpha,
\end{aligned}
$$

and the equations for the flow with boundary and initial conditions

$$
\begin{aligned}
& \frac{\partial u}{\partial t}=\frac{1}{r} \frac{\partial}{\partial r}\left(r \frac{\partial u}{\partial r}\right)+K^{2} \psi \cos (\omega t), \\
& u(r, t)=0, \quad r=\alpha, \\
& u(r, t)=0, \quad r=1, \\
& u(r, t)=0, \quad t=0 .
\end{aligned}
$$

Here $K^{2}=\kappa^{2} R^{2}, \psi_{\mathrm{i}}^{*}=z_{\mathrm{v}} e \psi_{\mathrm{i}} / k_{\mathrm{b}} T$ and $\psi_{\mathrm{o}}^{*}=z_{\mathrm{v}} e \psi_{\mathrm{o}} / k_{\mathrm{b}} T$ are normalized wall potentials.

\section{Analytical solutions}

The general solution of (7) has the form

$$
\psi(r)=A_{1} I_{0}(K r)+B_{1} K_{0}(K r)
$$

where $A_{1}$ and $B_{1}$ are undetermined constants, $I_{0}(r)$ and $K_{0}(r)$ are the modified Bessel functions of the first and second kind of order zero, respectively. Considering the boundary conditions (8) and (9), we have

$$
\psi(r)=\psi_{\mathrm{o}}^{*}\left[A I_{0}(K r)+B K_{0}(K r)\right]
$$

and the constants $A$ and $B$ are

$$
A=\frac{K_{0}(K \alpha)-\beta K_{0}(K)}{I_{0}(K) K_{0}(K \alpha)-I_{0}(K \alpha) K_{0}(K)}
$$

and

$$
B=\frac{I_{0}(K \alpha)-\beta I_{0}(K)}{K_{0}(K) I_{0}(K \alpha)-K_{0}(K \alpha) I_{0}(K)} .
$$

Here $\beta=\psi_{\mathrm{i}} / \psi_{\mathrm{o}}$ is defined as the ratio of the zeta potentials of the inner wall to that of the outer wall.

We now consider the solution of the governing equation (10); here it is not convenient to use the classical method of separation of variables because of the nonhomogeneities of the master governing equation (10). It is for this reason that we consider the integraltransform technique, and this method provides a systematic, efficient, and straightforward approach for the solution of both homogeneous and nonhomogeneous, steady-state, and time-dependent initial and boundary-value problems. 
The integral-transform pair in the $r$ variable for the function $T(r, t)$ is defined as [13]

$$
\begin{aligned}
& \tilde{T}\left(\lambda_{m}, t\right)=\int_{\alpha}^{1} r R_{0}\left(\lambda_{m}, r\right) T(r, t) d r, \\
& T(r, t)=\sum_{m=1}^{\infty} \frac{R_{0}\left(\lambda_{m}, r\right)}{N\left(\lambda_{m}\right)} \tilde{T}\left(\lambda_{m}, t\right),
\end{aligned}
$$

where

$$
\begin{aligned}
& R_{0}\left(\lambda_{m}, r\right)=J_{0}\left(\lambda_{m} r\right) Y_{0}\left(\lambda_{m}\right)-J_{0}\left(\lambda_{m}\right) Y_{0}\left(\lambda_{m} r\right), \\
& \frac{1}{N\left(\lambda_{m}\right)}=\frac{\pi^{2}}{2} \frac{\lambda_{m}^{2} J_{0}^{2}\left(\alpha \lambda_{m}\right)}{J_{0}^{2}\left(\alpha \lambda_{m}\right)-J_{0}^{2}\left(\lambda_{m}\right)},
\end{aligned}
$$

and $\lambda_{m}$ is the $m$ th positive root of $R_{0}\left(\lambda_{m}, \alpha\right)=0$.

Applying the above integral transform (16) to (7)-(13) yields

$$
\begin{aligned}
& \tilde{\psi}\left(\lambda_{m}\right)=\frac{2 \psi_{\mathrm{o}}^{*}}{\pi\left(\lambda_{m}^{2}+K^{2}\right)}\left(1-\beta \frac{J_{0}\left(\lambda_{m}\right)}{J_{0}\left(\alpha \lambda_{m}\right)}\right), \\
& \frac{d \tilde{u}}{d t}=-\lambda_{m}^{2} \tilde{u}+K^{2} \tilde{\psi} \cos (\omega t), \\
& \tilde{u}\left(\lambda_{m}, t\right)=0, \quad t=0 .
\end{aligned}
$$

Equation (21) is an ordinary differential equation with initial condition (22), and its solution can be given directly as

$$
\tilde{u}\left(\lambda_{m}, t\right)=K^{2} \tilde{\psi}\left(\lambda_{m}\right)\left[\frac{\sin \left(\omega t+\Phi_{m}\right)}{\sqrt{\lambda_{m}^{4}+\omega^{2}}}-\frac{\lambda_{m}^{2}}{\lambda_{m}^{4}+\omega^{2}} e^{-\lambda_{m}^{2} t}\right]
$$

where $\Phi_{m}=\arctan \left(\lambda_{m}^{2} / \omega\right)<\pi / 2$ is the phase difference or the phase shift, and $\tilde{\psi}\left(\lambda_{m}\right)$ can be obtained from (2) with the help of the aforementioned integral transform (16). Then, substituting $\tilde{\psi}\left(\lambda_{m}\right)$ into Eq. (23) yields the distribution of the velocity in the capillary,

$$
\begin{aligned}
u(r, t)= & K^{2} \sum_{m=1}^{\infty} \frac{\pi \lambda_{m}^{2} J_{0}^{2}\left(\alpha \lambda_{m}\right)\left[J_{0}\left(\lambda_{m} r\right) Y_{0}\left(\lambda_{m}\right)-J_{0}\left(\lambda_{m}\right) Y_{0}\left(\lambda_{m} r\right)\right]}{\left.\left(\lambda_{m}^{2}+K^{2}\right) J_{0}^{2}\left(\alpha \lambda_{m}\right)-J_{0}^{2}\left(\lambda_{m}\right)\right]} \\
& \times\left(\psi_{\mathrm{o}}^{*}-\psi_{\mathrm{i}}^{*} \frac{J_{0}\left(\lambda_{m}\right)}{J_{0}\left(\alpha \lambda_{m}\right)}\right)\left[\frac{\sin \left(\omega t+\Phi_{m}\right)}{\sqrt{\lambda_{m}^{4}+\omega^{2}}}-\frac{\lambda_{m}^{2}}{\lambda_{m}^{4}+\omega^{2}} e^{-\lambda_{m}^{2} t}\right] .
\end{aligned}
$$

\section{Results and discussion}

\subsection{The generation of the flow}

From the expression (24), we find that the velocity field of electro-osmotic flow in the capillary generated by the external applied electric field is not time periodic. In particular, the distribution of the velocity $u(r, \varphi, t)$ can be written as a sum of a time-dependent oscillating part $u_{1}(r, t)$ and a time-dependent generating part $u_{2}(r, t)$ :

$$
u(r, t)=u_{1}(r, t)-u_{2}(r, t)
$$


Table 1 First five roots of $R_{0}\left(\lambda_{m}, \alpha\right)=0$

\begin{tabular}{llllll}
\hline $\boldsymbol{\alpha}$ & $\boldsymbol{\lambda}_{\mathbf{1}}$ & \multicolumn{1}{c}{$\boldsymbol{\lambda}_{\mathbf{2}}$} & $\boldsymbol{\lambda}_{\mathbf{3}}$ & $\boldsymbol{\lambda}_{\mathbf{4}}$ & \multicolumn{1}{l}{$\boldsymbol{\lambda}_{\mathbf{5}}$} \\
\hline 0.1 & 3.3139 & 6.8576 & 10.3774 & 13.8864 & 17.3896 \\
0.2 & 3.8160 & 7.7855 & 11.7321 & 15.6702 & 19.6042 \\
0.3 & 4.4124 & 8.9328 & 13.4341 & 17.9292 & 22.4216 \\
0.4 & 5.1831 & 10.4432 & 15.6884 & 20.9292 & 26.1681 \\
0.5 & 6.2461 & 12.5469 & 18.8364 & 25.1228 & 31.4080 \\
\hline
\end{tabular}

where

$$
u_{1}(r, t)=\sum_{m=1}^{\infty} \frac{2 \psi_{\mathrm{o}}^{*} K^{2} R_{0}\left(\lambda_{m}, r\right)}{\pi N\left(\lambda_{m}\right)\left(\lambda_{m}^{2}+K^{2}\right)}\left(1-\beta \frac{J_{0}\left(\lambda_{m}\right)}{J_{0}\left(\alpha \lambda_{m}\right)}\right) \frac{\sin \left(\omega t+\Phi_{m}\right)}{\sqrt{\lambda_{m}^{4}+\omega^{2}}}
$$

and

$$
u_{2}(r, t)=\sum_{m=1}^{\infty} \frac{2 \psi_{\mathrm{o}}^{*} K^{2} R_{0}\left(\lambda_{m}, r\right)}{\pi N\left(\lambda_{m}\right)\left(\lambda_{m}^{2}+K^{2}\right)}\left(1-\beta \frac{J_{0}\left(\lambda_{m}\right)}{J_{0}\left(\alpha \lambda_{m}\right)}\right) \frac{\lambda_{m}^{2}}{\lambda_{m}^{4}+\omega^{2}} e^{-\lambda_{m}^{2} t}
$$

Here $R_{0}\left(\lambda_{m}, r\right)$ and $N\left(\lambda_{m}\right)$ are defined by (18) and (19), respectively.

The first five roots of $R_{0}\left(\lambda_{m}, \alpha\right)=0$ for different $\alpha$ are listed in Table 1 . It can be seen that the minimum of $\lambda_{m}$ increases with increasing $\alpha$. When $\alpha=0.1$, for the minimum of $\lambda_{m}$ we have $\lambda=\min \left\{\lambda_{m}\right\} \simeq 3.3139$, and $e^{-\lambda^{2}} \simeq 1.7 \times 10^{-5}$. As a result, we can draw the conclusion that the generating part of the solution (24) will tend to zero in a very short time, which results in the electro-osmotic flow reaching a steady 'periodic' state. Additionally, it is worth pointing out that the increasing frequency of the applied external electric field accelerates the generation of flow in the microannulus.

In the sense of the above discussion, the generating part can also be called the transient part. In other words, the electro-osmotic flow generated by the AC electric field is quasi-periodic. In spite of this, the generating part of the solution is very important for the researcher in this field, since it explains both the characteristics of electro-osmotic flow and the practical applications due to rapid development of the biochip technology [14]. Furthermore, in a study of the stability of a colloidal system, Overbeek [15] pointed out that the relaxation time for surface charges (about $10^{-6}$ to $10^{4} \mathrm{~s}$ ) and the time scale for Brownian coagulation (about $10^{-7}$ to $10^{-5} \mathrm{~s}$ ) are very different; the aggregation of colloidal particles may occur earlier than the equilibrium of the electrical conditions near a surface. In these cases, the steady-state analysis on the electrical condition near a charged surface is unrealistic, and an extension of the conventional treatment to a temporal description is inevitable, and this is the significance of the present study.

\subsection{Special cases}

In particular, when $\omega \rightarrow 0$, i.e., $E(t)=E_{0} H(t)$, where $H(t)$ is the Heaviside step function,

$$
\lim _{\omega \rightarrow 0} \arctan \frac{\lambda^{2}}{\omega}=\frac{\pi}{2}
$$

which yields

$$
\lim _{\omega \rightarrow 0} \sin \left(\omega t+\Phi_{m}\right)=1
$$


Then we get the distribution of velocity profile when the applied external electric field remains constant from time $t=0$, i.e., the electric field follows a step-change:

$$
\begin{aligned}
u(r, t)= & K^{2} \sum_{m=1}^{\infty} \frac{\pi \lambda_{m}^{2} J_{0}^{2}\left(\alpha \lambda_{m}\right)\left[J_{0}\left(\lambda_{m} r\right) Y_{0}\left(\lambda_{m}\right)-J_{0}\left(\lambda_{m}\right) Y_{0}\left(\lambda_{m} r\right)\right]}{\left.\left(\lambda_{m}^{2}+K^{2}\right) J_{0}^{2}\left(\alpha \lambda_{m}\right)-J_{0}^{2}\left(\lambda_{m}\right)\right]} \\
& \times\left(\psi_{\mathrm{o}}^{*}-\psi_{\mathrm{i}}^{*} \frac{J_{0}\left(\lambda_{m}\right)}{J_{0}\left(\alpha \lambda_{m}\right)}\right)\left[1-e^{-\lambda_{m}^{2} t}\right]
\end{aligned}
$$

When $\beta=1$, then we have $\psi_{\mathrm{o}}^{*}=\psi_{\mathrm{i}}^{*}$, i.e., the inner wall and outer one have the same zeta potential,

$$
\begin{aligned}
u(r, t)= & \psi_{\mathrm{o}}^{*} K^{2} \sum_{m=1}^{\infty} \frac{\pi \lambda_{m}^{2} J_{0}\left(\alpha \lambda_{m}\right) R_{0}\left(\lambda_{m}, r\right)}{\left(\lambda_{m}^{2}+K^{2}\right)\left[J_{0}\left(\alpha \lambda_{m}\right)+J_{0}\left(\lambda_{m}\right)\right]} \\
& \times\left[\frac{\sin \left(\omega t+\Phi_{m}\right)}{\sqrt{\lambda_{m}^{4}+\omega^{2}}}-\frac{\lambda_{m}^{2}}{\lambda_{m}^{4}+\omega^{2}} e^{-\lambda_{m}^{2} t}\right] .
\end{aligned}
$$

When $\beta=0, \psi_{i}^{*}=0$, i.e., the inner wall is not charged, then the solution of velocity (24) reduces to

$$
\begin{aligned}
u(r, t)= & \psi_{\mathrm{o}}^{*} K^{2} \sum_{m=1}^{\infty} \frac{\pi \lambda_{m}^{2} J_{0}^{2}\left(\alpha \lambda_{m}\right)\left[J_{0}\left(\lambda_{m} r\right) Y_{0}\left(\lambda_{m}\right)-J_{0}\left(\lambda_{m}\right) Y_{0}\left(\lambda_{m} r\right)\right]}{\left(\lambda_{m}^{2}+K^{2}\right)\left[J_{0}^{2}\left(\alpha \lambda_{m}\right)-J_{0}^{2}\left(\lambda_{m}\right)\right]} \\
& \times\left[\frac{\sin \left(\omega t+\Phi_{m}\right)}{\sqrt{\lambda_{m}^{4}+\omega^{2}}}-\frac{\lambda_{m}^{2}}{\lambda_{m}^{4}+\omega^{2}} e^{-\lambda_{m}^{2} t}\right] .
\end{aligned}
$$

\subsection{Effects of $K$ on velocity profiles and flow rates}

For given values $\alpha=0.3, \omega=10, \psi_{\mathrm{o}}=2$, and $\beta=1$, plots of the normalized velocity $u(r, t) / U_{\mathrm{eo}}$ as a function of the non-dimensional radius $r / R$ for selected values of time $t$ and for four different values of the electrokinetic width $K$ are shown in Figure 2. It is clear from this figure that the flow in the microannulus is similar to general pipe flow for small $K$ as shown in the figure when $K=50$, because the EDL is thicker for small $K$. When the value of $K$ is large enough, the velocity of the flow increases with increasing electrokinetic width $K$, and this phenomenon can also be proved analytically from the expression (24), where the term $K^{2} /\left(\lambda_{m}^{2}+K^{2}\right)$ is included. The same conclusion is true for the flow rate of fluid, which can be obtained by integrating Eq. (24),

$$
\begin{aligned}
Q(t)= & K^{2} \sum_{m=1}^{\infty} \frac{2 \pi^{2} \lambda_{m}^{2} J_{0}^{2}\left(\alpha \lambda_{m}\right)}{\left.\left(\lambda_{m}^{2}+K^{2}\right) J_{0}^{2}\left(\alpha \lambda_{m}\right)-J_{0}^{2}\left(\lambda_{m}\right)\right]}\left(\psi_{\mathrm{o}}^{*}-\psi_{\mathrm{i}}^{*} \frac{J_{0}\left(\lambda_{m}\right)}{J_{0}\left(\alpha \lambda_{m}\right)}\right) \\
& \times\left\{Y_{0}\left(\lambda_{m}\right)\left[J_{1}\left(\lambda_{m}\right) \alpha J_{1}\left(\alpha \lambda_{m}\right)\right]-J_{0}\left(\lambda_{m}\right)\left[Y_{1}\left(\lambda_{m}\right)-\alpha Y_{1}\left(\alpha \lambda_{m}\right)\right]\right\} \\
& \times\left[\frac{\sin \left(\omega t+\Phi_{m}\right)}{\sqrt{\lambda_{m}^{4}+\omega^{2}}}-\frac{\lambda_{m}^{2}}{\lambda_{m}^{4}+\omega^{2}} e^{-\lambda_{m}^{2} t}\right] .
\end{aligned}
$$

To demonstrate the effect of $K$ on the flow rate, plots of the flow rate as a function of $t$ using Eq. (33), for selected values of $K$ and $\omega$, are displayed in Figure 3. The physical mechanism for the above phenomena is that a large $K$ indicates a thinner EDL, then the region of bulk flow (mass flow) becomes wider, which results in more mass of fluid through the pipe during the same period. 

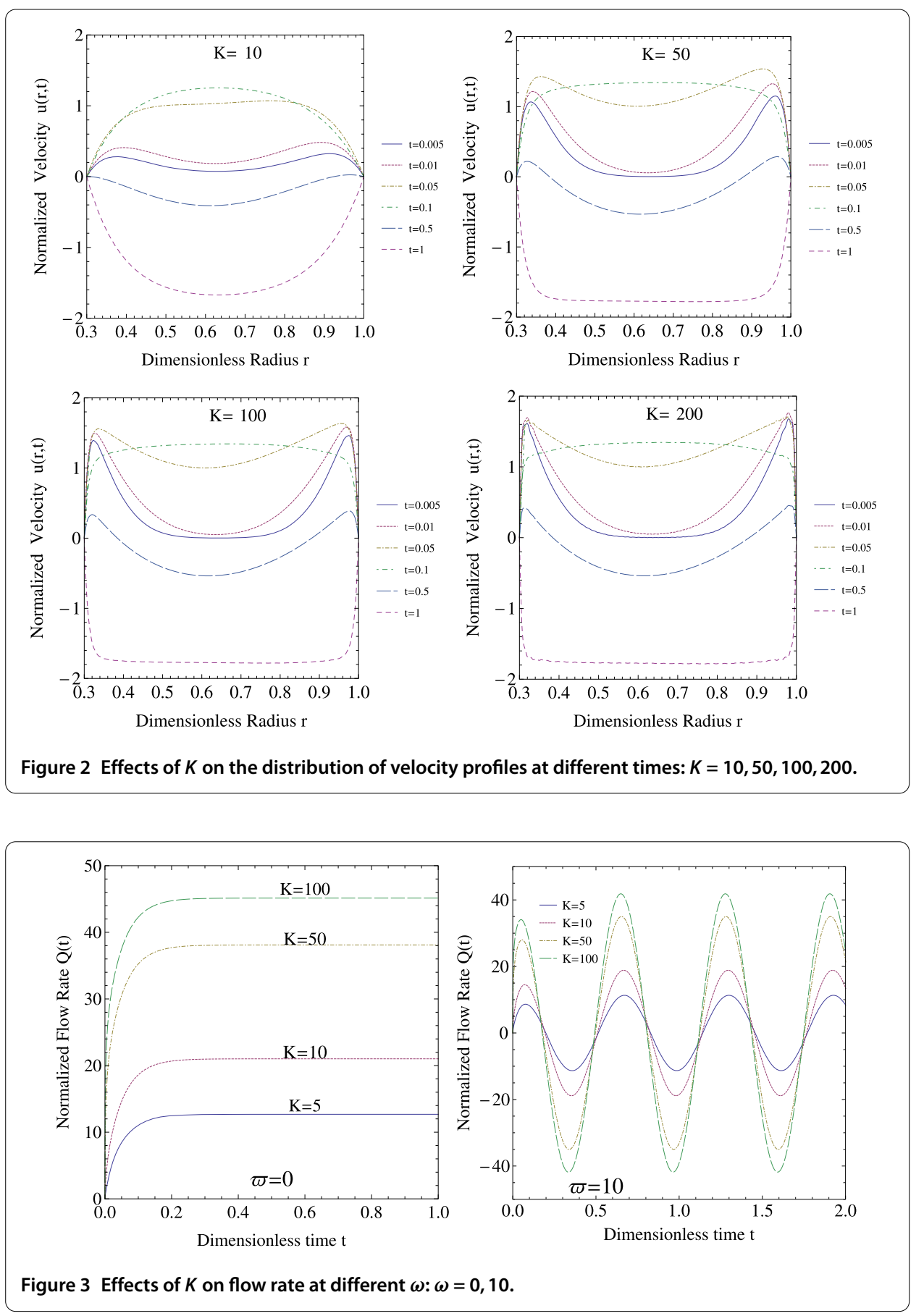

Additionally, some authors drew the conclusion that the flow rate is proportional to the cross-sectional area of the channel for large $K$, and the flow rate is quadratic as $K^{2}$ in the leading-order behavior for small $K$ [16-18]; it is noteworthy that this conclusion is incorrect. In this research, the authors discussed the asymptotic expansion of $K^{2} /\left(\lambda_{m}^{2}+K^{2}\right)$ for large and small $K$, respectively, with the help of series summation formulas. Unfortunately, they ignored the fact that $\left\{\lambda_{m}\right\}$ is a monotonically increasing infinite subsequence. In other words, for a given $K$, no matter how large it is, there exists a natural number $N$ such that if $m \geq N$, then $\lambda_{m} \geq K$. 
On the other hand, from Figure 3 and Eqs. (26)-(27), it is important to note that because the transient part of the velocity solution decays in a short time, if the researchers consider the electro-osmotic flow of fluids for time $t>1$, the time-dependent oscillating part $u_{1}(r, t)$ can be used as a good approximation for simplicity of computation.

\subsection{Effects of $\omega$ on velocity profiles}

Figure 4 indicates the axial velocity distributions along the axial direction during the period of transient response from $t=0$ to $t=1$ for different values of $\omega$. For given $\alpha=0.3$, $K=100, \psi_{\mathrm{o}}=2$, and $\beta=1$, the oscillation of flow is enhanced by the increasing frequency of the applied external electric field. However, the mean velocity of flow decreases as $\omega$ increases from $\omega=10$ to $\omega=1,000$. An interesting phenomenon is found: there is almost no flow in the areas far away from the EDL when $\omega$ is large enough, as in the case of $\omega=1,000$.

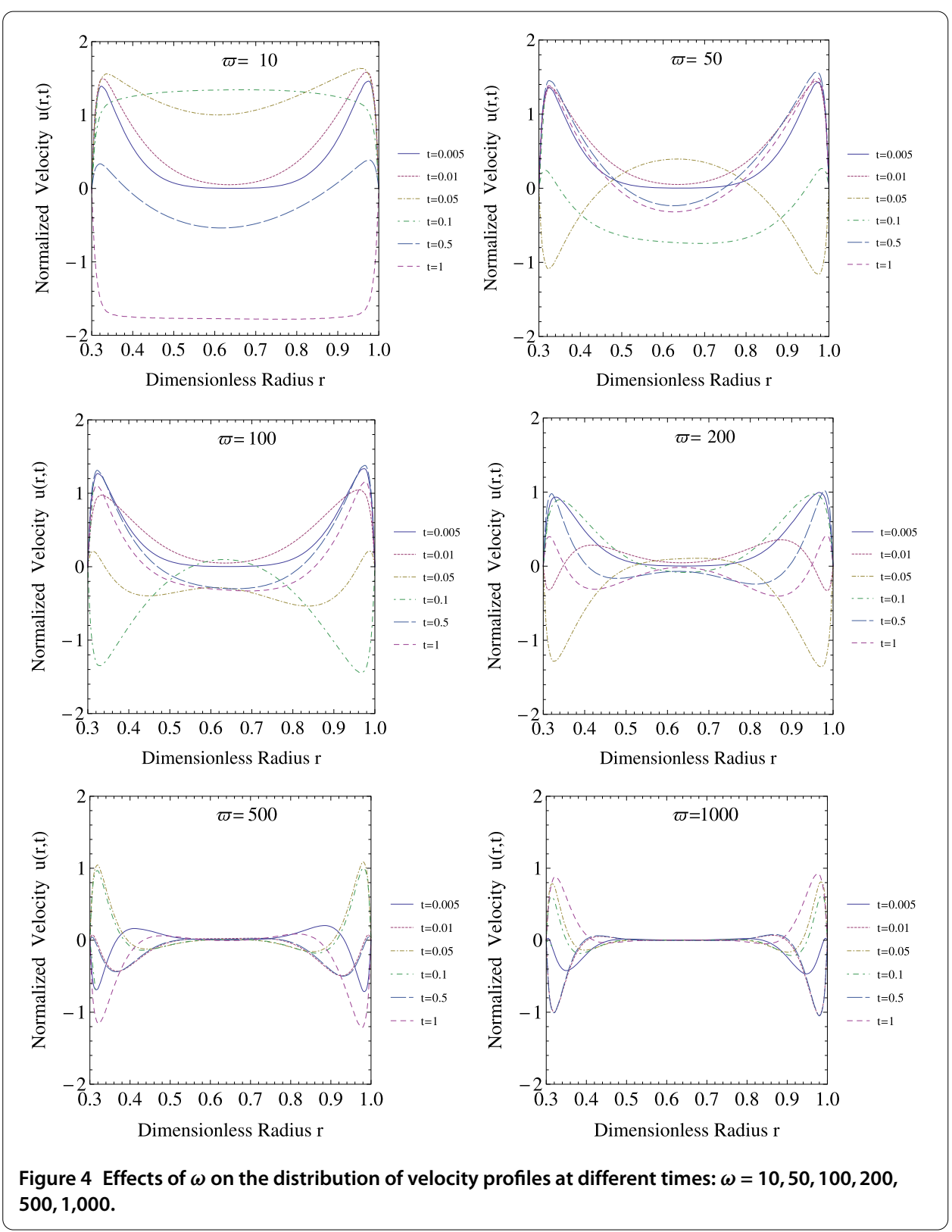


Figure 5 Effects of $\omega$ on the flow rate at fixed times $t=0.1$.

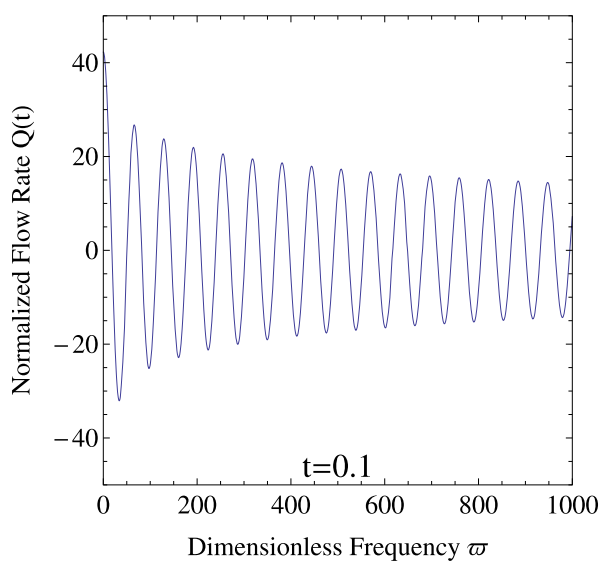

As the frequency of external electric field increases, the flow rate gets closer to zero, and it keeps its oscillation, as shown in Figure 5, which is obtained from (33) as a function of $\omega$ at fixed $t=0.1$. The physical interpretation is that the large frequency AC electric field makes the ions in the fluid oscillate around an equilibrium position. Mathematically and analytically, the increasing $\omega$ decreases the term in Eq. (24),

$$
\frac{\sin \left(\omega t+\Phi_{m}\right)}{\sqrt{\lambda_{m}^{4}+\omega^{2}}}-\frac{\lambda_{m}^{2}}{\lambda_{m}^{4}+\omega^{2}} e^{-\lambda_{m}^{2} t}
$$

as a result, the velocity reliably decreases with increasing frequency of the applied external electric field. Away from the walls of the microannulus, the EDL can be divided into the compact layer and the diffuse double layer. Within the diffuse layer, the motion of the ions is subject to the zeta potential of the EDL. Then we have the conclusion that the main contribution to the flow rate is the zeta potential when the frequency of the applied AC electric field is large enough.

Additionally, the effects of $\omega$ on the normalized flow rate and phase shift are shown in Figure 6 for given $K=5$. With the increasing frequency of the applied external electrical field, there appears to be a decrease of the phase shift between voltage and flow. In fact, according to Eq. (23), $\phi_{m}$ mathematically tends to be zero for large enough $\omega$. At the same time, Figure 6 shows the increasing frequency decreases the flow rate $Q(t)$, which is consistent with the results shown in Figure 4 and Figure 5.

\subsection{Effects of wall zeta potential ratio $\beta$ on velocity profiles}

Electro-osmotic flow is the result of the interaction between the applied external electric field and the electric double layer, so it is necessary to discuss the effect of the wall's zeta potential ratio $\beta$ of the inner to the outer cylinder on the velocity profiles. For each fixed time $t$, a sequence of $u(r, t)$ curves for different values of $\beta$ is shown in Figure 7. From the figure, it can be seen that the direction of the flow is directly correlated with the polarity of the charged channel wall. When $\beta<0$, flow reversal is observed, which is caused by an adverse zeta potentials on the walls. When $\beta>0$, the velocity of flow increases with increasing $\beta$, this can also be proved theoretically with the help of the average velocity in 

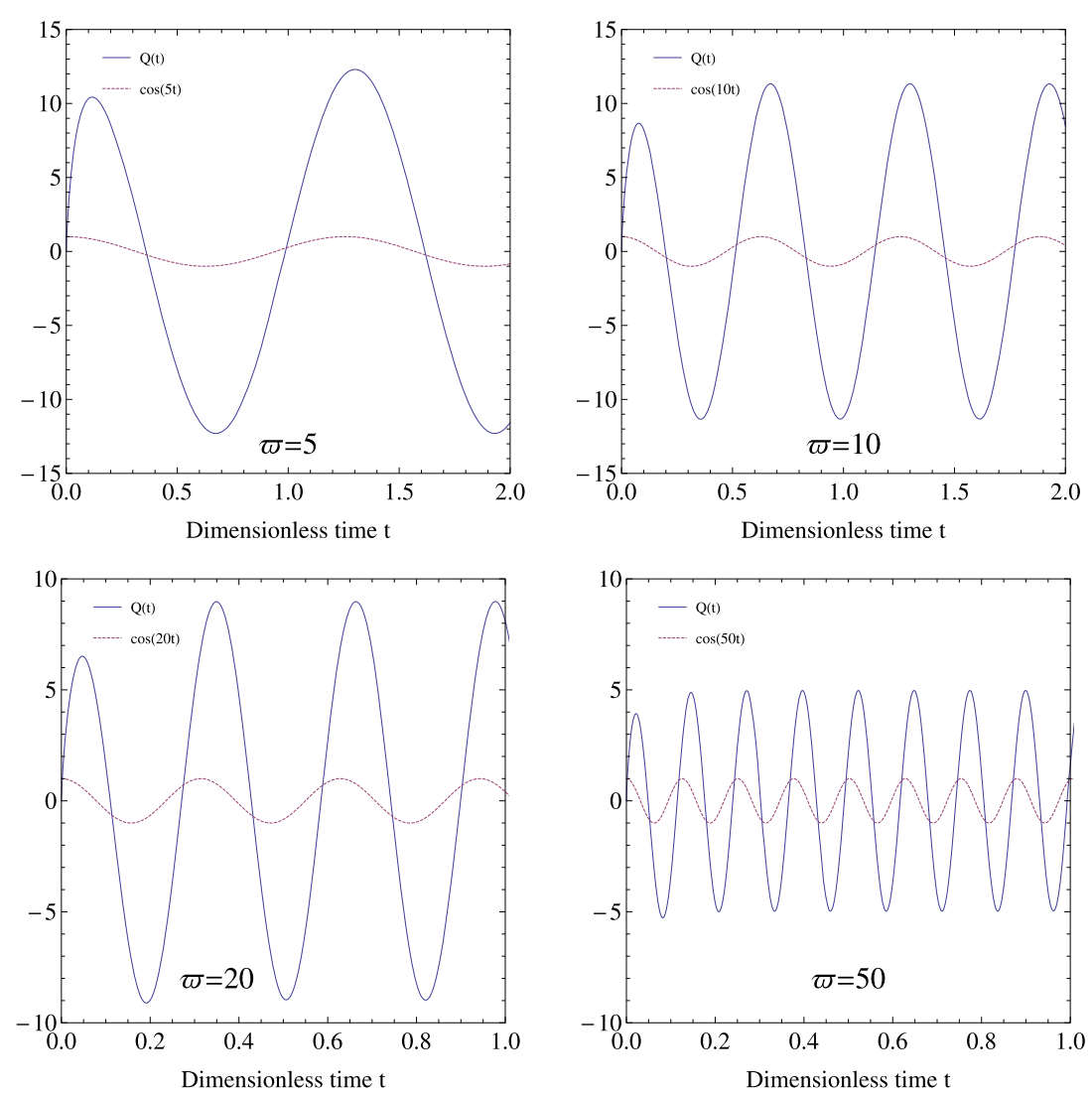

Figure 6 Effects of $\omega$ on the flow rate and phase shift: $\omega=5,10,20,50$.

the microannulus, i.e., $\langle u(t)\rangle=Q(t) / 2 \pi$, and the following relationship is obtained:

$$
\langle u(t)\rangle \sim 1-\beta \frac{J_{0}\left(\lambda_{m}\right)}{J_{0}\left(\alpha \lambda_{m}\right)} .
$$

\section{Summary and conclusion}

It has not been a accurate task to find the analytic solutions for time-periodic electroosmotic flow (AC electro-osmotic). In present research, we pointed out and corrected the errors in the published research articles in this field, and we obtained an analytical solutions of distribution of velocity profiles and flow rates for time-periodic electroosmotic flow in microannulus. With the help of numerical plots, our analysis has resulted some remarks. The velocity field of the electro-osmosis flow consists of two parts, a timedependent oscillating part and a time-dependent generating or transient one. The transient part tends to zero very fast with the increasing time. The electro-osmosis flow driven by an alternating electric field is not periodic in time, but quasi-periodic. There is a phase shift between applied external electric field and the electro-osmotic flow, which is only dependent of the frequency of external electric field, and it is less than $\pi / 2$. The increasing values of $K$ decrease the average velocity and flow rate, particularly, there is almost no flow in the region far from the walls when $\omega$ is large enough. Additionally, the increasing frequency of applied external electric field accelerates the generation of flow in the microannulus. The corresponding physical interpretation is given. 


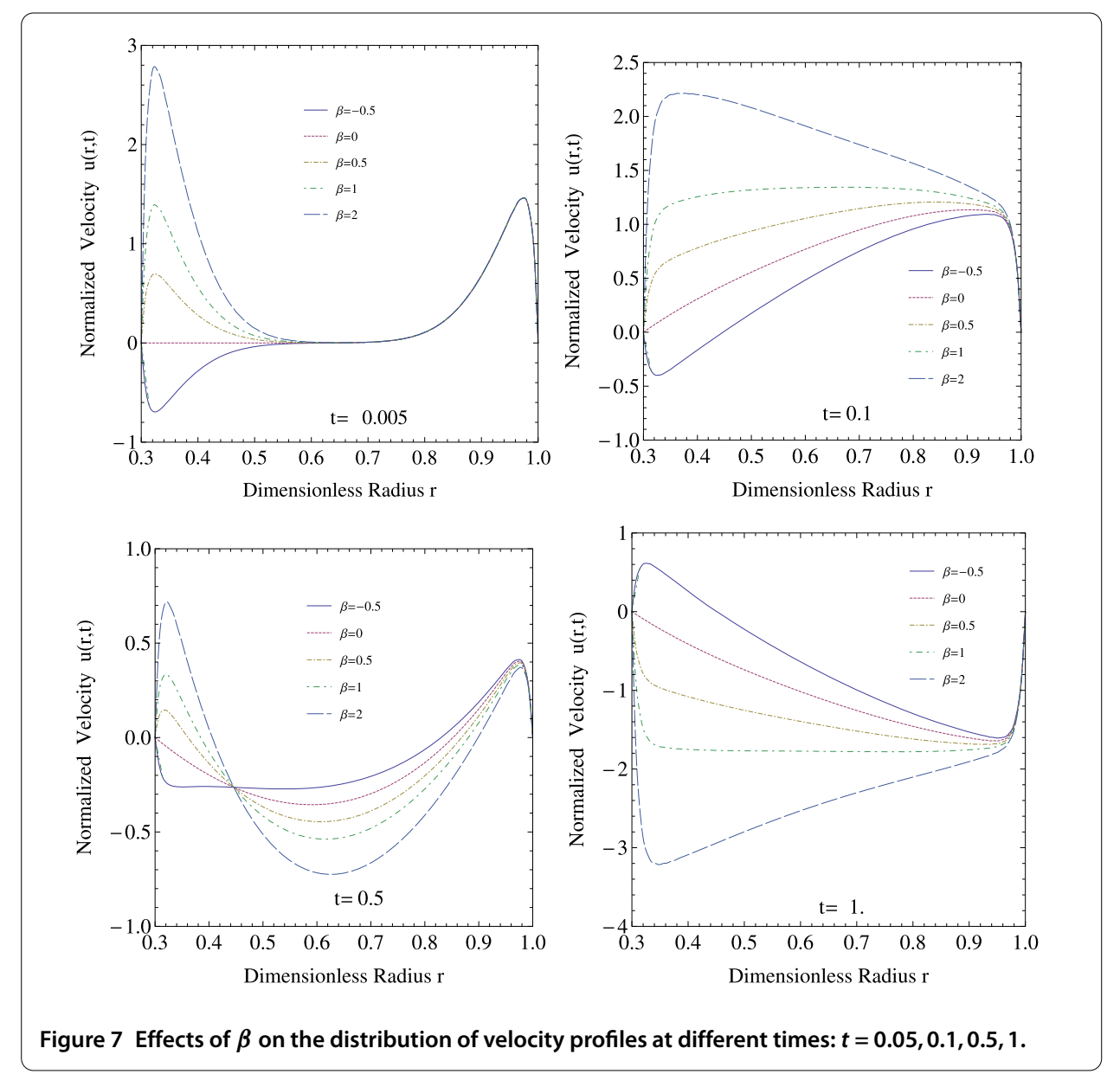

\section{Competing interests}

The authors declare that they have no competing interests.

\section{Authors' contributions}

All authors contributed equally to the writing of this paper. All authors read and approved the final manuscript.

\section{Acknowledgements}

This work is supported by the National Natural Science Foundation of China (Nos. 11002083, 51279093), the National Basic Research Program of China (2013CB0360000).

Received: 9 June 2014 Accepted: 9 September 2014 Published online: 26 September 2014

\section{References}

1. Effenhauser, CS, Manz, A, Widmer, HM: Manipulation of sample fractions on a capillary electrophoresis chip. Anal. Chem. 67, 2284-2287 (1995)

2. Raymond, DE, Manz, A, Widmer, HM: Continuous separation of high molecular weight compounds using a microliter volume free-flow electrophoresis microstructure. Anal. Chem. 68, 2515-2522 (1996)

3. Harrison, DJ, Manz, A, Fan, ZH, Ludi, H, Widmer, HM: Capillary electrophoresis and sample injection systems integrated on a planar glass chip. Anal. Chem. 64, 1926-1932 (1992)

4. Ramsey, RS, Ramsey, JM: Generating electrospray from microchip devices using electroosmotic pumping. Anal. Chem. 69, 1174-1178 (1997)

5. Dutta, P, Beskok, A: Analytical solution of time periodic electroosmotic flows: analogies to Stokes' second problem. Anal. Chem. 73, 5097-5102 (2001)

6. Xuan, X, Li, D: Electroosmotic flow in microchannels with arbitrary geometry and arbitrary distribution of wall charge. J. Colloid Interface Sci. 289, 291-303 (2005)

7. Jian, Y, Yang, L, Liu, Q: Time periodic electro-osmotic flow through a microannulus. Phys. Fluids 22, 042001 (2010)

8. Bao, LP, Jian, YJ, Chang, L, Su, J, Zhang, HY, Liu, QS: Time periodic electroosmotic flow of the generalized Maxwell fluids in a semicircular microchannel. Commun. Theor. Phys. 59, 615-622 (2013)

9. Nayak, AK: An analysis of steady/unsteady electroosmotic flows through charged cylindrical nano-channels. Theor. Comput. Fluid Dyn. (2013). doi:10.1007/s00162-013-0295-0 
10. Luo, WJ: Transient electroosmotic flow induced by DC or AC electric fields in a curved microtube. J. Colloid Interface Sci. 278, 497-507 (2004)

11. Erickson, D, Li, D: Analysis of alternating current electroosmotic flows in a rectangular microchannel. Langmuir 19 5421-5430 (2003)

12. Shilov, VN, Delgado, AV, González-Caballero, F, Horno, J, López-García, JJ, Grosse, C: Polarization of the electrical double layer. Time evolution after application of an electric field. J. Colloid Interface Sci. 232, 141-148 (2000)

13. Özisik, MN: Heat Conduction, 2nd edn. Wiley, New York (1993)

14. Wong, PK, Chen, C, Wang, T, Ho, C: Electrokinetic bioprocessor for concentrating cells and molecules. Anal. Chem. 76, 6908-6914 (2004)

15. Overbeek, JTG: Recent developments in the understanding of colloid stability. J. Colloid Interface Sci. 58, 408-422 (1977)

16. Wang, CY, Liu, YH, Chang, CC: Analytical solution of electro-osmotic flow in a semicircular microchannel. Phys. Fluids 20, $063105(2008)$

17. Wang, CY, Chang, CC: EOF using the Ritz method: application to superelliptic microchannels. Electrophoresis 28 , 3296-3301 (2007)

18. Chang, CC, Kuo, CY, Wang, CY: Unsteady electroosmosis in a microchannel with Poisson-Boltzmann charge distribution. Electrophoresis 32, 3341-3347 (2011)

doi:10.1186/s13661-014-0215-2

Cite this article as: Wang and Zhao: Closed-form solutions of transient electro-osmotic flow driven by AC

electric field in a microannulus. Boundary Value Problems 2014 2014:215.

Submit your manuscript to a SpringerOpen ${ }^{\odot}$ journal and benefit from:

- Convenient online submission

Rigorous peer review

- Immediate publication on acceptance

- Open access: articles freely available online

- High visibility within the field

- Retaining the copyright to your article

Submit your next manuscript at $>$ springeropen.com 